\title{
Association of gastro-oesophageal reflux and chronic rhinosinusitis: systematic review and meta-analysis*
}

\author{
Stewart R. Leason', Henry P. Barham², Gretchen Oakley',3, Janet Rimmer ${ }^{4,5}$, \\ John M. DelGaudio ${ }^{6}$, Jenna M. Christensen', Raymond Sacks ${ }^{1,3,7}$, \\ Richard J. Harvey ${ }^{1,3}$ \\ ' Rhinology and Skull Base Research Group, St Vincent's Centre for Applied Medical Research, University of New South Wales, \\ Sydney, Australia \\ ${ }^{2}$ Department of Otolaryngology Head and Neck Surgery, Louisiana State University, New Orleans, LO, USA \\ ${ }^{3}$ Faculty of Medicine and Health Sciences, Macquarie University, Sydney, Australia \\ ${ }^{4}$ St Vincent's Clinic, St Vincent's Hospital, Sydney, Australia \\ ${ }^{5}$ The Woolcock Institute, University of Sydney, Sydney, Australia \\ ${ }^{6}$ Division of Rhinology and Sinus Surgery, Department of Otolaryngology, Emory University School of Medicine, Atlanta, GA, USA \\ ${ }^{7}$ Department of Otolaryngology, Head and Neck Surgery, Concord General Hospital, University of Sydney, Sydney, Australia
}

Rhinology 55: 3-16, 2017

https:://doi.org/10.4193/Rhino16.177

*Received for publication:

May 24, 2016

Accepted: November 9, 2016

\begin{abstract}
Introduction: Gastro-oesophageal reflux disease (GORD) has been implicated in the development of chronic rhinosinusitis (CRS). The association of GORD with CRS is systematically assessed from the medical literature.

Methodology: Embase and MEDLINE were searched using a comprehensive strategy limited to English language and Human subjects. Any study with original data on the experimental, diagnostic, treatment or prognostic association of CRS with GORD was included. Studies without a control group, case reports and review articles were excluded.

Results: The search returned 958 records, with an additional 10 found from bibliographic lists; this produced 32 studies. The included studies ( $n=32$ ) consisted of studies reporting pathogenic factors $(n=20)$, epidemiological association ( $n=8)$, prognostic interactions ( $n=3$ ), and a combination of these outcomes $(n=1)$. Potential pathogenic roles for GORD in CRS were supported; CRS subjects had greater prevalence of intranasal Helicobacter pylori and acid reflux than subjects without CRS. CRS is more prevalent in GORD sufferers than those without GORD. Evidence is conflicting for GORD as a factor in CRS treatment failure.
\end{abstract}

Conclusion: The results support a significant association of GORD with CRS. Physicians should be cognizant of the potential for acid and non-acid reflux as a driving factor in CRS.

Key words: sinusitis, gastroesophageal reflux, Helicobacter pylori, review, meta-analysis

\section{Introduction}

Chronic rhinosinusitis (CRS) is a multifactorial disease. Reports of CRS prevalence vary between studies. Sixteen percent have been found to self-report chronic 'sinus trouble'(1), while population studies have found prevalence of $5-15 \%{ }^{(2,3)}$ and assessment of medical records in the US has found $2 \%{ }^{(4)}$.

Gastro-oesophageal reflux disease (GORD) is defined by the reflux of gastric contents, resulting in troublesome symptoms or complications ${ }^{(5)}$. Prevalence in the general population is estimated at $10-20 \%{ }^{(6)}$. Symptoms of GORD include heartburn and regurgitation, as well as extra-oesophageal complaints. A clear aetiological role for GORD has been identified in chronic cough, laryngeal complaints, asthma and dental erosions. In other conditions, such as CRS, any such role is putative. 
Multiple pathogenic mechanisms connecting GORD with CRS have been proposed and investigated. Furthermore, a role for anti-reflux treatment in recalcitrant CRS has been investigated and is often suggested in reviews. This can involve lifestyle changes, such as weight loss, smoking cessation, dietary and sleep modification; medical therapy with proton pump inhibitors (PPIs), histamine receptor antagonists (H2RAs), antacids and prokinetics; or surgery ${ }^{(7)}$.

The precise role of GORD in CRS remains highly ambiguous; despite frequent discussion in the literature, there is no systematic review of the evidence. This study aims to systematically review the literature published in this field and assess the evidence for a role of GORD in CRS disease.

\section{Materials and methods}

\section{Eligibility criteria}

Only studies of GORD related outcomes in CRS populations, or vice versa, were included. Studies with all reasonable definitions of CRS and GORD were included; no age or comorbidity restrictions were applied. Case-control studies, crossover studies, cohort studies and randomised controlled trials (RCTs) were included. Only manuscripts published in English were eligible; publications with no original data were excluded, as were case series, case reports, in vitro and animal studies.

\section{Information sources}

A systematic electronic search was performed until January 26th 2015 on the Embase (1974-) and Medline (1946-) databases. A search strategy was designed for each database (Table 1) to identify all studies of CRS and GORD, including studies with GORD investigations or treatments where its presence may not have been explicitly stated.

Review for studies missed by the search strategy was performed by scanning the bibliography of each study that discussed the association of GORD with CRS. This process was extended to articles with no original data that were not included in the final analysis. One review article could not be assessed because it was unavailable electronically and was no longer possessed by its publisher.

\section{Study selection}

The search results underwent unblinded review by two of the authors (SRL and HPB), being selected according to the predetermined criteria. Initial screening was upon title review, with brief abstract review if there was uncertainty. The remaining selection underwent stringent abstract review, with discussion between reviewers if uncertain about relevance of individual studies. The full texts of the subsequent selection were analysed, with study exclusion if not relevant. Case series and studies of rhinitis were included in the full text analysis, however they were subsequently excluded if CRS was not also assessed in a quantifiable fashion with an appropriate control group. Three different study types were identified and those for inclusion were grouped accordingly. These were studies of:

1. Pathogenic or aetiological role of GORD in CRS, assessing the presence of $H$. pylori, extra-gastric reflux or altered neurological pathways in subjects with CRS, or assessing nasal mucosa abnormalities in GORD

2. Epidemiological association between GORD and CRS populations

3. Prognostic interaction between GORD and CRS

\section{Data extraction}

A standardised datasheet was used for data extraction. Two authors (SRL and GO) recorded the following variables: study type, number of subjects in each population, population definitions, diagnostic criteria, exclusion criteria, outcomes, measures of assessment and criteria for their interpretation, and assessment site when relevant.

CRS and GORD were both dichotomously defined as present or absent. When studies reported multiple separate groups with either condition, based on type or severity, these were collapsed into one group for the current report.

When the presence of $H$. pylori was assessed, DNA, urease and immunohistochemical testing for $H$. pylori antigens were preferred over immunoassays for $\mathrm{H}$. pylori antibodies. If more than one of the former tests was performed, subjects were only considered to have $H$. pylori if at least two were positive.

If $\mathrm{pH}$ testing included multiple probes, results from all extraoesophageal sites and the most proximal oesophageal site were reported. Oesophageal reflux was considered significant using the most stringent criteria used in each study, so that the current review would only include subjects with the strongest possible evidence of significant reflux. Extra-oesophageal reflux was considered significant for subjects who had any true reflux episode with $\mathrm{pH} \leq 4$, since the pharynx lacks the protective mechanisms against reflux that exist in the oesophagus. Extra-oesophageal reflux episodes with $\mathrm{pH}>4$ were recorded separately for the current review to explore the role of weakly acidic reflux.

\section{Risk of bias}

No formal bias assessment was performed, as the final systematic review and meta-analysis included no randomised control trials and only one cohort study.

\section{Statistical analysis}

Case control studies were analysed using a fixed effects model (Mantel-Haenszel method) to obtain an odds ratio (OR). This was performed when comparable populations and outcomes were reported, with means reported for both groups. These were reported as OR with $95 \%$ confidence interval. 


\begin{tabular}{|c|c|c|c|}
\hline $\begin{array}{l}\text { Population } \\
\text { (CRS) }\end{array}$ & $\begin{array}{l}\text { Outcome } 1 \\
\text { (GORD - condition) }\end{array}$ & $\begin{array}{l}\text { Outcome } 2 \\
\text { (GORD - evaluation finding) }\end{array}$ & $\begin{array}{l}\text { Outcome } 3 \\
\text { (GORD - management) }\end{array}$ \\
\hline $\begin{array}{l}\text { \#1 exp sinusitis/ } \\
\text { \#2 (rhinosinusit* OR nasosinusit*).tw. } \\
\text { OR (sinusit* OR pansinusit* } \\
\text { OR ethmoidit* OR antrit* OR sphenoi- } \\
\text { dit*).tw. } \\
\text { \#3 (inflamm* adj3 sinus*) } \\
\text { \#4 exp rhinitis/ } \\
\text { \#5 (\#1 OR \#2 OR \#3 OR \#4) }\end{array}$ & $\begin{array}{l}\text { \#6 exp esophagus/ } \\
\text { \#7 (esophag* OR oesophag*).tw. } \\
\text { \#8 exp gastroesophageal reflux/ } \\
\text { \#9 (gastroesophageal reflux OR gastro } \\
\text { esophageal reflux OR gastro oesopha- } \\
\text { geal reflux).tw. } \\
\text { \#10 (gord OR gerd OR ger).tw. } \\
\text { \#11 duodenogastric reflux/ } \\
\text { \#12 (duodenogastric adj2 reflux).tw. } \\
\text { \#13 bile reflux/ } \\
\text { \# } 14 \text { (bile adj2 reflux).tw. } \\
\text { \#15 (acid adj5 reflux).tw. } \\
\text { \#16 (gastric acid secret* OR stomach } \\
\text { acid secret*).tw. } \\
\text { \#17 exp peptic ulcer/ } \\
\text { \#18 (gastric eros* OR stomach eros*). } \\
\text { tw. } \\
\text { \#19 heartburn/ } \\
\text { \#20 (heartburn OR indigestion).tw. } \\
\text { \#21 exp esophagitis/ } \\
\text { \#22 (esophagitis OR oesophagitis).tw. } \\
\text { \#23 (reflux esophagitis OR reflux } \\
\text { oesophagitis).tw. } \\
\text { \#24 (reflux laryngitis OR laryn- } \\
\text { geal reflux OR pharyngeal reflux OR } \\
\text { laryngopharyngeal reflux OR laryngo- } \\
\text { pharyngeal reflux OR Ipr OR posterior } \\
\text { laryngitis).tw. } \\
\text { \#25 (extraesophageal reflux OR } \\
\text { extraoesophageal reflux OR extra eso- } \\
\text { phageal reflux OR extra oesophageal } \\
\text { reflux).tw. } \\
\text { \#26 (heterotopic gastric mucosal } \\
\text { patch).tw. } \\
\text { \#27 (cervical inlet patch).tw. } \\
\text { \#28 (low* sphincter* pressur*).tw. } \\
\text { \#29 (les).tw. } \\
\text { \#30 gastric emptying/ } \\
\text { \#31 gastroparesis/ } \\
\text { \#32 exp gastritis/ } \\
\text { \#33 (gastr* empt* disorder*).tw. } \\
\text { \#34 (stomach empt* disorder*).tw. } \\
\text { \#35 exp dyspepsia/ } \\
\text { \#36 (dyspep*).tw. } \\
\text { \#37 eructation/ } \\
\text { \#38 (eructat* OR burp* OR belch*).tw. } \\
\text { \#39 (regurgitat*).tw. } \\
\text { \#40 hernia, hiatal/ } \\
\text { \#41 (hiat* hernia).tw. } \\
\text { \#42 (\#6 OR \#7 OR \#8 OR \#9 OR \#10 OR } \\
\text { \#11OR \#12 OR \#13 OR \#14 OR \#15 OR } \\
\text { \#16 OR \#17 OR \#18 OR \#19 OR \#20 OR } \\
\text { \# } 21 \text { OR \#22 OR \#23 OR \#24 OR \#25 } \\
\text { OR \#26 OR \#27 OR \#28 OR \#29 OR \#30 } \\
\text { OR \#31 OR \#32 OR \#33 OR \#34 OR \#35 } \\
\text { OR \#36 OR \#37 OR \#38 OR \#39 OR \#40 } \\
\text { OR \#41) }\end{array}$ & 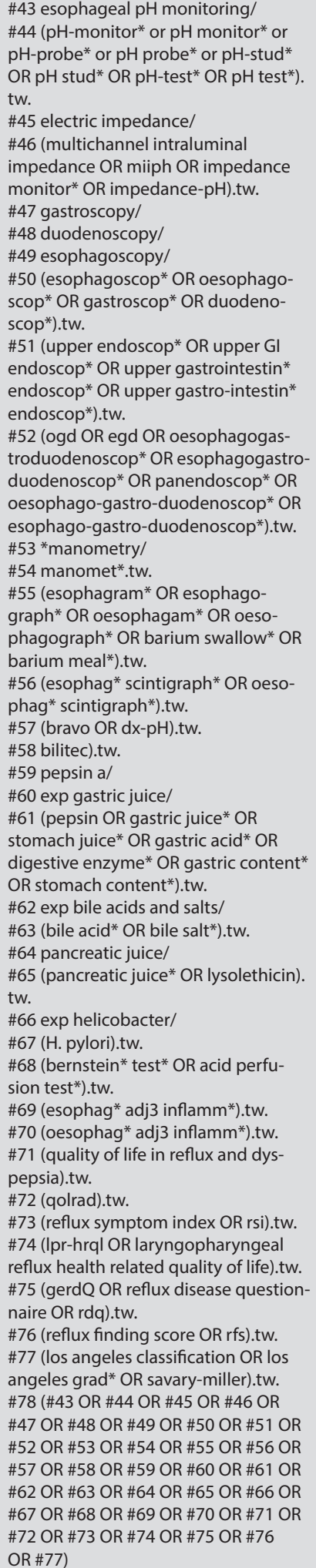 & $\begin{array}{l}\text { \#79 exp proton pump/ } \\
\text { \#80 proton pump inhibitors/ } \\
\text { \#81 exp anti-ulcer agents/ } \\
\text { \#82 (proton pump inhibitor*).tw. } \\
\text { \#83 (ppi*).tw. } \\
\text { \#84 (omeprazole OR esomeprazole } \\
\text { OR lansoprazole OR lanzoprazole OR } \\
\text { dexlansoproazole OR pantoprazole OR } \\
\text { rabeprazole).tw. } \\
\text { \#85 exp histamine H2 antagonists/ } \\
\text { \#86 (histamine adj3 h2 adj3 antago- } \\
\text { nist*).tw. } \\
\text { \#87 (h2ra*).tw. } \\
\text { \#88 (cimetidine OR famotidine OR } \\
\text { nizatidine OR ranitidine OR metiamide } \\
\text { OR burimamide).tw. } \\
\text { \#89 exp anti-ulcer agents/ } \\
\text { \#90 (anti ulcer* agent* OR antiulcer* } \\
\text { agent* OR antireflux* agent* OR } \\
\text { anti reflux* agent* OR antireflux* } \\
\text { treatment* OR anti reflux* treatment* } \\
\text { OR antireflux* therapy* OR anti reflux* } \\
\text { therapy* OR antireflux* medication* } \\
\text { OR anti reflux* medication*).tw. } \\
\text { \# } 91 \text { (prokinetic*).tw. } \\
\text { \#92 (domperidone OR metocloprami- } \\
\text { de OR cisapride OR erythromycin).tw. } \\
\text { \#93 exp antacids/ } \\
\text { \#94 (antacid*).tw. } \\
\text { \#95 fundoplication/ } \\
\text { \#96 (nissen OR rossetti OR toupet OR } \\
\text { lind OR watson OR besley).tw. } \\
\text { \#97 (partial* fundoplication*).tw. } \\
\text { \#98 (laparoscop* fundoplication*).tw. } \\
\text { \#99 (lifestyle modification* OR diet* } \\
\text { modification*).tw. } \\
\text { \#100 (\#79 OR \#80 OR \#81 OR \#82 OR } \\
\text { \#83 OR \#84 OR \#85 OR \#86 OR \#87 OR } \\
\text { \#88 OR \#89 OR \#90 OR \#91 OR \#92 OR } \\
\text { \#93 OR \#94 OR \#95 OR \#96 OR \#97 OR } \\
\text { \#98 OR \#99) } \\
\text { Final } \\
\text { \#101 (\#42 OR \#77 OR \#100) } \\
\text { \#102 (\#5 AND \#101) }\end{array}$ \\
\hline
\end{tabular}




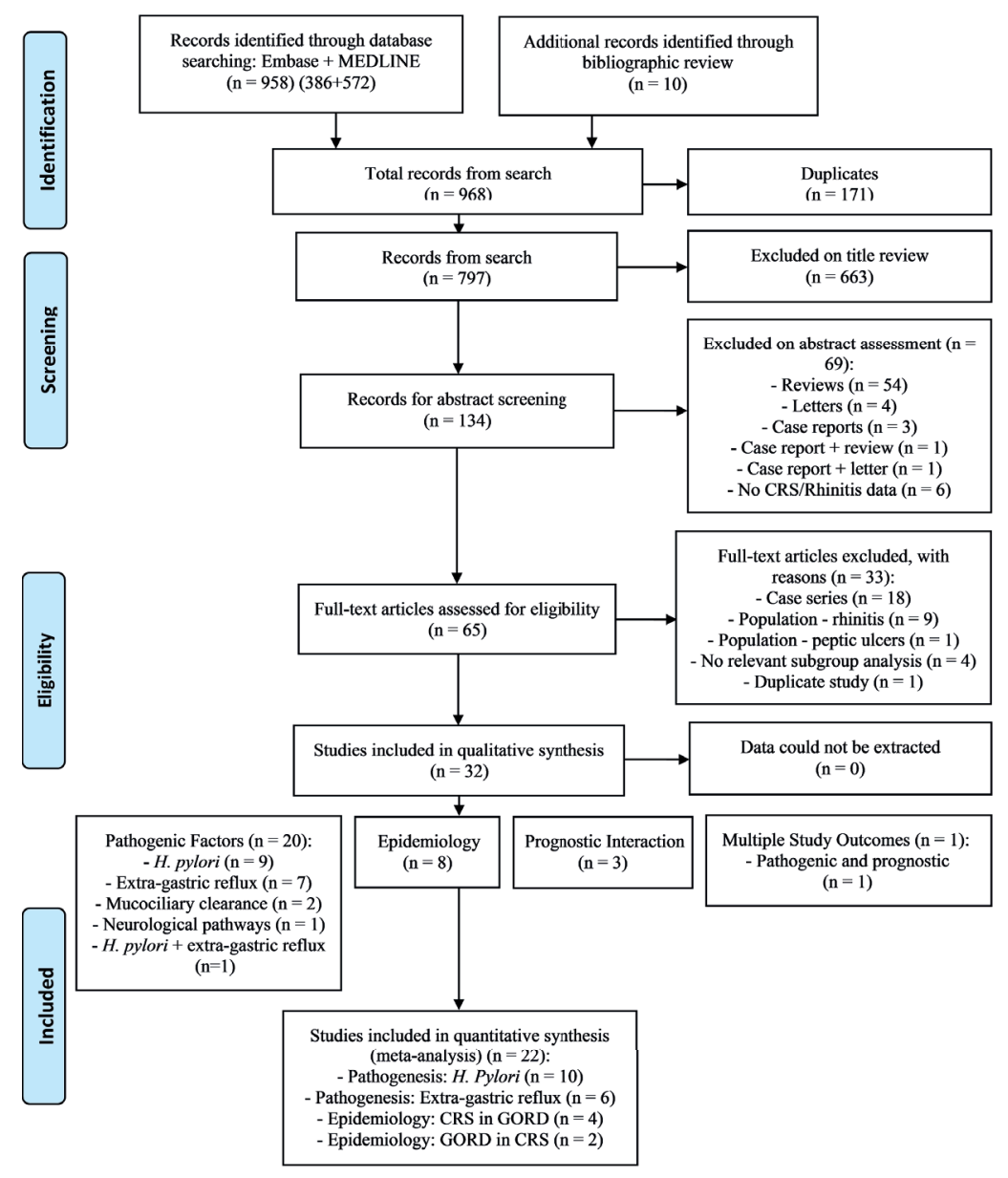

Figure 1. PRISMA flow diagram of study selection.

\section{Results}

Search results

The search strategy found 958 records; bibliographic review found another 10 articles. This was reduced to 797 after the removal of 171 duplicates. Title and abstract review found that 134 discussed the association between GORD and CRS or rhinitis. Sixty three of these were excluded because they did not include any original data and six did not contain relevant data. On full text analysis, another 33 articles were excluded. Eighteen excluded publications were case series, studying CRS response to GORD treatment ${ }^{(8-14)}$, GORD pathogenesis in CRS $(8,12,13,15-19)$, and epidemiological association between GORD and CRS (20-25). Nine excluded publications only studied rhinitis, one only studied peptic ulcers, four had no relevant subgroup analysis and one presented data that was also published in another study.

The final review was of 32 publications (Figure 1). Two publications each contained two different and relevant studies, which were split for analysis. The characteristics of the included studies are shown in Table 2-4. There was one prospective cohort study and 33 case-control studies within these 32 publications.
Twenty five studies were of adults, one was of children and eight included both or did not specify the age range. There were a total of 255,323 participants.

\section{Study aims}

Studies were grouped as follows:

- Twenty one studies aimed to examine the presence of factors pathogenically linked to CRS which included the presence of $H$. pylori, extra-gastric reflux, neurological pathways and nasal mucosal abnormalities in GORD

- Eight studies examined an epidemiological association between GORD and CRS

- Four examined a prognostic interaction between GORD and CRS

\section{Definitions of CRS and GORD}

The groups were variably defined. Seven studies used international consensus definitions for CRS. Four studies used diagnosis of CRS as recorded in medical files. Others studies defined CRS using a variable combination of symptomatology, duration and investigative findings. No definition was provided for CRS in two 
Table 2. Characteristics of included studies - studies of factors pathogenically linked to chronic rhinosinusitis (CRS).

\begin{tabular}{|c|c|c|c|c|c|}
\hline Study & Design & $\mathbf{N}$ & Population & Outcome & Mode of Assessment \\
\hline \multirow[t]{2}{*}{$\begin{array}{l}\text { Burduk, } \\
2011^{(53)}\end{array}$} & $\mathrm{CC}^{\mathrm{a}}$ & 20 & Adults with CRSwNPb & $\begin{array}{l}\text { Presence of intranasal } \\
\text { H. pylori }\end{array}$ & $\begin{array}{l}\text { ure } A \text { and cagA genes (amplification PCR }{ }^{c} \text { with gel electropho- } \\
\text { resis) }\end{array}$ \\
\hline & & 10 & Normal nasal mucosa & & \\
\hline \multirow[t]{2}{*}{$\begin{array}{l}\text { Cvorovic, } \\
2008^{(26)}\end{array}$} & CC & 23 & Adults with CRSwNP & $\begin{array}{l}\text { Presence of intranasal } \\
\text { H. pylori }\end{array}$ & $\begin{array}{l}\text { Urease (CLOd test) and H. pylori cells (histochemical analysis } \\
\text { with Giemsa staining) }\end{array}$ \\
\hline & & 15 & Normal nasal mucosa & & \\
\hline \multirow[t]{2}{*}{$\begin{array}{l}\text { Ozyurt, } \\
2009^{(54)}\end{array}$} & CC & 32 & Adults with CRSwNP & $\begin{array}{l}\text { Presence of intranasal } \\
\text { H. pylori }\end{array}$ & ure $C$ gene and cagA gene (real-time PCR) \\
\hline & & 27 & Normal nasal mucosa & & \\
\hline \multirow[t]{2}{*}{$\begin{array}{l}\text { Dinis, } \\
2006^{(32)}\end{array}$} & CC & 15 & $\begin{array}{l}\text { Adults with CRS } \\
\text { (unspecified) }\end{array}$ & $\begin{array}{l}\text { Presence of intranasal } \\
\text { H. pylori }\end{array}$ & ureA gene (amplification PCR with gel electrophoresis) \\
\hline & & 5 & Normal nasal mucosa & & \\
\hline \multirow[t]{2}{*}{$\begin{array}{l}\text { Kim, } \\
2007^{(55)}\end{array}$} & CC & 48 & $\begin{array}{l}\text { Subjects with CRSe } \\
\text { (unspecified) }\end{array}$ & $\begin{array}{l}\text { Presence of intranasal } \\
\text { H. pylori }\end{array}$ & $\begin{array}{l}\text { Urease (CLO test) and H. pylori cells (immunohistochemical } \\
\text { staining) }\end{array}$ \\
\hline & & 29 & Normal nasal mucosa & & \\
\hline \multirow[t]{2}{*}{$\begin{array}{l}\text { Ozdek, } \\
2003^{(28)}\end{array}$} & CC & 12 & $\begin{array}{l}\text { Adults with CRS } \\
\text { (unspecified) }\end{array}$ & $\begin{array}{l}\text { Presence of intranasal } \\
\text { H. pylori }\end{array}$ & $\begin{array}{l}\text { 16S ribosomal RNA gene (with primers Hp1 \& Hp3, then pri- } \\
\text { mers } \mathrm{Hp} 1 \text { \& Hp2 by nested PCR with gel electrophoresis) }\end{array}$ \\
\hline & & 13 & Normal nasal mucosa & & \\
\hline \multirow[t]{2}{*}{$\begin{array}{l}\text { Koc, } \\
2004\end{array}$} & CC & 30 & $\begin{array}{l}\text { Subjects with nasal } \\
\text { polyps (unspecified) }\end{array}$ & $\begin{array}{l}\text { Presence of intranasal } \\
\text { H. pylori }\end{array}$ & $\begin{array}{l}\text { Anti-H. pylori lgG in serum (ELISAg) or H. pylori cells in nasal } \\
\text { tissue (immunohistochemical staining) }\end{array}$ \\
\hline & & 20 & Normal nasal mucosa & & \\
\hline \multirow[t]{2}{*}{$\begin{array}{l}\text { Nemati, } \\
2012^{(29)}\end{array}$} & CC & 25 & $\begin{array}{l}\text { Subjects with nasal } \\
\text { polyps (unspecified) }\end{array}$ & $\begin{array}{l}\text { Presence of intranasal } \\
\text { H. pylori }\end{array}$ & $\begin{array}{l}\text { ureC gene (amplification PCR with gel electrophoresis), urease } \\
\text { (CLO test) or H. pylori cells in nasal tissue (culture) }\end{array}$ \\
\hline & & 25 & Normal nasal mucosa & & \\
\hline \multirow[t]{2}{*}{$\begin{array}{l}\text { Ozcan, } \\
2009^{(56)}\end{array}$} & CC & 25 & $\begin{array}{l}\text { Adults with nasal } \\
\text { polyps (unspecified) }\end{array}$ & $\begin{array}{l}\text { Presence of intranasal } \\
\text { H. pylori }\end{array}$ & $\begin{array}{l}\text { Urease (CLO test) or } H \text {. pylori cells in nasal tissue (immunohis- } \\
\text { tochemical staining); anti-H. pylori lgG or IgA } A^{h} \text { in serum (ELISA) }\end{array}$ \\
\hline & & 14 & Normal nasal mucosa & & \\
\hline \multirow[t]{2}{*}{$\begin{array}{l}\text { Vceva, } \\
2012^{(46)}\end{array}$} & CC & 35 & $\begin{array}{l}\text { Adults with nasal } \\
\text { polyps (unspecified) }\end{array}$ & $\begin{array}{l}\text { Presence of intranasal } \\
\text { H. pylori }\end{array}$ & $\begin{array}{l}\text { H. pylori DNA in nasal tissue (real-time PCR) and anti-H. pylori } \\
\lg (\operatorname{lgG} \text { or } \lg A) \text { in serum (ELISA) }\end{array}$ \\
\hline & & 30 & Normal nasal mucosa & & \\
\hline \multirow[t]{3}{*}{$\begin{array}{l}\text { DelGaudio, } \\
2005^{(39)}\end{array}$} & CC & 38 & $\begin{array}{l}\text { Adults with CRS } \\
\text { (unspecified) }\end{array}$ & Presence of GORDk & $\begin{array}{l}24 \text { hour } \mathrm{pH} \text { study (with nasopharyngeal } \mathrm{pH}<4 \text { and }<5 \text {; oeso- } \\
\text { phageal } \mathrm{pH}<4 \text { for }>4.0 \% \text { of study) }\end{array}$ \\
\hline & & 10 & $\begin{array}{l}\text { CRS (unspecified) } \\
\text { cured by FESS }\end{array}$ & & \\
\hline & & 20 & No history of CRS & & \\
\hline \multirow[t]{3}{*}{$\begin{array}{l}\text { Ulualp, } \\
\text { 1999b (57) }\end{array}$} & CC & 6 & $\begin{array}{l}\text { Adults with CRS (un- } \\
\text { specified) and LPR }\end{array}$ & Presence of GORD & 24 hour pH study (with hypopharyngeal pH < 4) \\
\hline & & 12 & $\begin{array}{l}\text { CRS (unspecified) } \\
\text { alone }\end{array}$ & & \\
\hline & & 34 & No CRS & & \\
\hline \multirow[t]{2}{*}{$\begin{array}{l}\text { Ulualp, } \\
\text { 1999a }{ }^{(58)}\end{array}$} & CC & 11 & $\begin{array}{l}\text { Adults with CRS } \\
\text { (unspecified) }\end{array}$ & Presence of GORD & $\begin{array}{l}24 \text { hour pH study (with hypopharyngeal and oesophageal } \\
\mathrm{pH}<4 \text { ) }\end{array}$ \\
\hline & & 11 & No CRS or GORD & & \\
\hline \multirow[t]{2}{*}{$\begin{array}{l}\text { Ozmen, } \\
2008^{(59)}\end{array}$} & CC & 33 & $\begin{array}{l}\text { Subjects with CRS } \\
\text { (unspecified) }\end{array}$ & $\begin{array}{l}\text { Presence of GORD and } \\
\text { of intra-nasal pepsin }\end{array}$ & $\begin{array}{l}24 \text { hour } \mathrm{pH} \text { study (with hypopharyngeal } \mathrm{pH}<4 \text { ) and nasal } \\
\text { pepsin in saline lavage (by fluorometric assay) }\end{array}$ \\
\hline & & 20 & Normal nasal mucosa & & \\
\hline \multirow[t]{2}{*}{$\begin{array}{l}\text { Jecker, } \\
2006^{(30)}\end{array}$} & CC & 20 & Adults with CRSwNP & Presence of GORD & $\begin{array}{l}24 \text { hour pH study (by DeMeester score and total duration of } \\
\text { oesophageal reflux episodes) }\end{array}$ \\
\hline & & 20 & No CRS & & \\
\hline \multirow[t]{2}{*}{$\begin{array}{l}\text { Bhawana, } \\
2014^{(31)}\end{array}$} & CC & 50 & $\begin{array}{l}\text { Adults with CRS } \\
\text { (unspecified) }\end{array}$ & Intra-nasal pH & Single stable $\mathrm{pH}$ reading at middle meatus \\
\hline & & 50 & No CRS & & \\
\hline
\end{tabular}




\begin{tabular}{|c|c|c|c|c|c|}
\hline Study & Design & $\mathbf{N}$ & Population & Outcome & Mode of Assessment \\
\hline \multirow[t]{2}{*}{$\begin{array}{l}\text { Loehrl, } \\
2012^{(33)}\end{array}$} & $\mathrm{CC}$ & 5 & $\begin{array}{l}\text { Adults with CRS } \\
\text { (unspecified) }\end{array}$ & $\begin{array}{l}\text { Presence of intra-nasal } \\
\text { pepsin }\end{array}$ & $\begin{array}{l}\text { Nasal pepsin in saline lavage (by gel electrophoresis and } \\
\text { Western blot) }\end{array}$ \\
\hline & & 5 & No CRS or GORD & & \\
\hline \multirow[t]{2}{*}{$\begin{array}{l}\text { Dinis, } \\
2006^{(32)}\end{array}$} & $\mathrm{CC}$ & 15 & $\begin{array}{l}\text { Adults with CRS } \\
\text { (unspecified) }\end{array}$ & $\begin{array}{l}\text { Presence of intra-nasal } \\
\text { pepsin }\end{array}$ & $\begin{array}{l}\text { Nasal pepsin and pepsinogen I concentrations in nasal biopsy } \\
\text { (by chemiluminescent immunoassay) }\end{array}$ \\
\hline & & 5 & Normal nasal mucosa & & \\
\hline \multirow[t]{2}{*}{$\begin{array}{l}\text { Catalano, } \\
2004^{(61)}\end{array}$} & $\mathrm{CC}$ & 38 & $\begin{array}{l}\text { Subjects with CRS } \\
\text { (unspecified) }\end{array}$ & Presence of GORD & Oesophagitis on endoscopic visualisation and histopathology \\
\hline & & 117 & No CRS or GORD & & \\
\hline \multirow[t]{2}{*}{$\begin{array}{l}\text { Delehaye, } \\
2009^{(34)}\end{array}$} & $\mathrm{CC}$ & 37 & Adults with GORD & $\begin{array}{l}\text { Mucocilliary clearance } \\
\text { time and sinonasal } \\
\text { symptoms }\end{array}$ & Saccharine clearance time and SNOT-20' \\
\hline & & 13 & Adults with LPR ${ }^{m}$ & & \\
\hline \multirow[t]{2}{*}{$\begin{array}{l}\text { Durmus, } \\
2010^{(35)}\end{array}$} & $\mathrm{CC}$ & 50 & Adults with GORD & $\begin{array}{l}\text { Mucociliary clearance } \\
\text { time and change with } \\
\text { GORD treatment }\end{array}$ & $\begin{array}{l}\text { Saccharine clearance time (pre and post lansoprazole 30mg } \\
\text { BD for } 12 \text { weeks for GORD group) }\end{array}$ \\
\hline & & 30 & No GORD & & \\
\hline \multirow[t]{2}{*}{$\begin{array}{l}\text { Wong, } \\
2010^{(36)}\end{array}$} & Cohort & 10 & $\begin{array}{l}\text { Adults with no CRS or } \\
\text { GORD, oesophageal } \\
\text { acid infusion }\end{array}$ & $\begin{array}{l}\text { Nasal effects of oeso- } \\
\text { phageal reflux }\end{array}$ & $\begin{array}{l}\text { Nasal inspiratory peak flow (best of three), sinonasal } \\
\text { symptoms }\left(V A S^{n}\right) \text {, nasal mucus secretions (by fructose level in } \\
\text { nasal lavage) }\end{array}$ \\
\hline & & 10 & $\begin{array}{l}\text { No CRS or GORD, } \\
\text { oesophageal saline } \\
\text { infusion }\end{array}$ & & \\
\hline
\end{tabular}

a) CC: case-control; b) CRSwNP: chronic rhinosinusitis with nasal polyps; c) PCR: polymerase chain reaction; d) CLO: Campylobacter-like organism; e) CRS: chronic rhinosinusitis; f) IgG: immunoglobulin-G; g) ELISA: enzyme-linked immunosorbent assay; h) IgA: immunoglobulin-A; i) CS: case series; j) PCR-MPH: polymerase chain reaction with microplate hybdidisation; k) GORD: gastro-oesophageal reflux disease; I) SNOT-20: sino-nasal outcome test-20; m) LPR: laryngopharyngeal reflux; m) VAS: visual-analogue scale

studies.

Diagnosis of GORD was based on register in medical files in seven studies. In other studies, GORD was defined by symptoms or positive investigative findings, which included endoscopy, biopsy, pH studies and testing.

\section{Pathogenic factors}

These study findings are summarised in Table 5. The presence of H. pylori in sinonasal tissue was the outcome of 10 case-control studies, assessed using different microbiological tests. These studies included 265 subjects with CRS and 188 with normal nasal mucosa. Meta-analysis found an increased odds ratio (OR) of $H$. pylori in CRS (OR 2.88 [1.58-5.26]) overall, although not all studies found this association (Figure 2). H. pylori prevalence in CRS was $31.7 \%(84 / 265)$.

The presence of GORD was not assessed by six of these studies. GORD was assessed in three studies(26-28), which found that $87.5 \%$ (14/16) of subjects with intranasal H. pylori had GORD. Nemati ${ }^{(29)}$, who found no intranasal $H$. pylori in any subjects, had excluded GORD sufferers from the study.

Evidence of extra-gastric reflux in CRS was assessed through nine case-control studies. $\mathrm{pH}$ testing was employed by six of the studies, while nasal pepsin was assessed by three studies and oesophageal endoscopic and biopsy findings were used by one. $\mathrm{pH}$ testing was generally performed for 24 -hours, with differing probe sites and numbers.

Meta-analysis of six studies found reflux was more common in those with CRS (75/143) than without CRS (40/207) (OR 4.03 [2.37-6.86]) (Figure 3). This gave an overall finding of reflux among $52.4 \%$ of subjects with CRS.

Three other studies could not be included in meta-analysis. One of these found significantly more reflux in the subjects with CRS than without CRS, based on mean DeMeester scores ${ }^{(30)}$. Bhawana and Kumar ${ }^{(31)}$ found the middle meatus more alkaline with CRS ( $\mathrm{pH} 7.81 \pm 0.83$ ) than without $C R S$, although no subjects had GORD symptoms. Dinis and Subtil (32) found no difference in the pepsin and pepsinogen I concentrations in nasopharyngeal tissue of subjects with or without CRS, and concentrations were consistently lower than serum concentration. This was similar to the findings of Loehrl ${ }^{(33)}$, who did not detect pepsin in any nasopharyngeal biopsies, although it was present in nasopharyngeal aspirates of the same subjects.

Functional nasal mucosa differences in GORD subjects were assessed by mucociliary clearance time. One case-control study 
Table 3. Characteristics of included studies - epidemiological association between gastro-oesophageal reflux (GORD) and chronic rhinosinusitis (CRS).

\begin{tabular}{|c|c|c|c|c|c|}
\hline Study & Design & $\mathbf{N}$ & Population & Outcome & Mode of Assessment \\
\hline \multirow[t]{2}{*}{$\begin{array}{l}\text { Tan, } \\
2013{ }^{(62)}\end{array}$} & $\mathrm{CC}^{\mathrm{a}}$ & 595 & $\begin{array}{l}\text { Subjects with } \\
\text { CRSwNPb }\end{array}$ & $\begin{array}{l}\mathrm{GORD}^{c} \\
\text { prevalence }\end{array}$ & $\begin{array}{l}\text { Medical records (GPd, specialist, inpatient or ERe) (ICD-9-CM }{ }^{f} \text { GORD } \\
\text { code }(530.81))\end{array}$ \\
\hline & & $\begin{array}{l}7523 \\
8118\end{array}$ & $\begin{array}{l}\text { with CRSsNPg } \\
\text { without CRS }\end{array}$ & & \\
\hline \multirow[t]{2}{*}{$\begin{array}{l}\text { Sedaghat, } \\
2012^{(63)}\end{array}$} & CC & 24 & $\begin{array}{l}\text { Adults with } A R^{i} \\
\text { and subsequent } \\
\text { CRS (unspecified) }\end{array}$ & $\begin{array}{l}\text { GORD } \\
\text { prevalence }\end{array}$ & $\begin{array}{l}\text { Self-report to otolaryngologist, with specialist diagnosis and sup- } \\
\text { porting history (unspecified) }\end{array}$ \\
\hline & & 35 & with AR alone & & \\
\hline \multirow[t]{2}{*}{$\begin{array}{l}\text { Ruhl, } \\
2001^{(64)}\end{array}$} & CC & 537 & Adults with GORD & RSi incidence & $\begin{array}{l}\text { Medical records (inpatient) or death certificates (ICD-9-CM sinusitis } \\
\text { codes }(461,473) \text { ) }\end{array}$ \\
\hline & & 6391 & without GORD & & \\
\hline \multirow[t]{2}{*}{$\begin{array}{l}\text { Ruigomez, } \\
2004^{(37)}\end{array}$} & CC & 7159 & $\begin{array}{l}\text { Subjects with } \\
\text { GORD }\end{array}$ & RS incidence & $\begin{array}{l}\text { Medical records (GP) < } 1 \text { year after GORD diagnosis (criteria unspe- } \\
\text { cified) }\end{array}$ \\
\hline & & 10000 & without GORD & & \\
\hline \multirow[t]{2}{*}{$\begin{array}{l}\text { El-Serag, } \\
2001^{(65)}\end{array}$} & CC & 1980 & $\begin{array}{l}\text { Children with } \\
\text { GORD }\end{array}$ & RS prevalence & $\begin{array}{l}\text { Medical records (inpatient) (recorded sinus surgery or ICD-9-CM } \\
\text { sinusitis codes }(461.9,473) \text { ) }\end{array}$ \\
\hline & & 7920 & without GORD & & \\
\hline \multirow{2}{*}{$\begin{array}{l}\text { El-Serag, } \\
1997^{(66)}\end{array}$} & $\mathrm{CC}$ & 101366 & Adults with GORD & RS prevalence & Medical records (inpatient) (ICD-9-CM sinusitis codes (unspecified)) \\
\hline & & 101366 & without GORD & & \\
\hline \multirow[t]{2}{*}{$\begin{array}{l}\text { Katle, } \\
2012^{(38)}\end{array}$} & CC & 77 & $\begin{array}{l}\text { Subjects with } \\
\text { GORD }\end{array}$ & RS prevalence & Symptom questionnaire (by SNOT-20k score) \\
\hline & & 480 & $\begin{array}{l}\text { General } \\
\text { population }\end{array}$ & & \\
\hline \multirow[t]{2}{*}{$\begin{array}{l}\text { Theodoropou- } \\
\text { los, } 2001^{(49)}\end{array}$} & $\mathrm{CC}$ & 36 & Adults with GORD & RS prevalence & $\begin{array}{l}\text { Symptom questionnaire (with common nasal or sinus symptoms } \\
>4 \text { days/month) }\end{array}$ \\
\hline & & 74 & without GORD & & \\
\hline
\end{tabular}

a) CC: case-control; b) CRSwNP: chronic rhinosinusitis with nasal polyps; c) GORD: gastro-oesophageal reflux disease; d) GP: general practitioner; e) ER: emergency room; $f$ ) ICD-9-CM: International Classification of Diseases-9-Clinical Modification; g) CRSsNP: chronic rhinosinusitis without nasal polyps; h) CRS: chronic rhinosinusitis; i) AR: allergic rhinitis; j) RS: rhinosinusisits; k) SNOT-20: sino-nasal outcome test-20; I) CS: case series

Table 4. Characteristics of included studies - prognostic interaction between gastro-oesophageal reflux (GORD) and chronic rhinosinusitis (CRS).

\begin{tabular}{|c|c|c|c|c|c|c|}
\hline Study & Design & $\mathbf{N}$ & Population & Outcome & $\begin{array}{l}\text { Mode of } \\
\text { Assessment }\end{array}$ & Measurement System \\
\hline \multirow[t]{2}{*}{$\begin{array}{l}\text { DelGaudio, } \\
2005^{(39)}\end{array}$} & $\mathrm{CC}^{\mathrm{a}}$ & 38 & $\begin{array}{l}\text { Adults with CRS } \\
\text { (unspecified) }\end{array}$ & $\begin{array}{l}\text { Presence of } \\
\text { GORD }^{c}\end{array}$ & 24 hour pH study & $\begin{array}{l}\text { Any true nasopharyngeal reflux episode with } \\
\mathrm{pH}<4\end{array}$ \\
\hline & & $\begin{array}{l}10 \\
20\end{array}$ & $\begin{array}{l}\text { CRS (unspecified) } \\
\text { cured by FESS } \\
\text { No history of sinonasal } \\
\text { disease }\end{array}$ & & & \\
\hline \multirow[t]{2}{*}{$\begin{array}{l}\text { Chambers, } \\
1997^{(40)}\end{array}$} & CC & 42 & $\begin{array}{l}\text { Adults with CRS (un- } \\
\text { specified) }\end{array}$ & $\begin{array}{l}\text { Presence of } \\
\text { GORD }\end{array}$ & $\begin{array}{l}\text { Medical records } \\
\text { (otolaryngologist) }\end{array}$ & $\begin{array}{l}\text { Medicated symptomatic GORD (self-reported } \\
\text { or chart listed) }\end{array}$ \\
\hline & & 140 & $\begin{array}{l}\text { CRS (unspecified) } \\
\text { cured by FESS }\end{array}$ & & & \\
\hline \multirow[t]{2}{*}{$\begin{array}{l}\text { Deconde, } \\
2014^{(41)}\end{array}$} & CC & 72 & $\begin{array}{l}\text { Adults with CRS (un- } \\
\text { specified) and GORD }\end{array}$ & $\begin{array}{l}\text { Response } \\
\text { to FESS }\end{array}$ & $\begin{array}{l}\text { Subjective and } \\
\text { objective measures } \\
\text { of sinus disease }\end{array}$ & $\begin{array}{l}\text { Change in } \mathrm{QoL}^{\mathrm{e}} \text { scores (RSDIf, } \mathrm{SNOT}-22^{9} \text { and } \\
\text { CSS }{ }^{h} \text { ) or nasoendoscopic findings (Lund \& } \\
\text { Kennedy scoring system) }\end{array}$ \\
\hline & & 157 & $\begin{array}{l}\text { CRS (unspecified) } \\
\text { without GORD }\end{array}$ & & & \\
\hline \multirow[t]{2}{*}{$\begin{array}{l}\text { Jelavic, } \\
2012^{(42)}\end{array}$} & CC & 28 & $\begin{array}{l}\text { Adults with CRSwNPi } \\
\text { with } H \text {. pylori in nasal } \\
\text { polyps }\end{array}$ & $\begin{array}{l}\text { Response } \\
\text { to FESS }\end{array}$ & $\begin{array}{l}\text { Subjective and } \\
\text { objective measures } \\
\text { of sinus disease }\end{array}$ & $\begin{array}{l}\text { Change in symptom severity and frequency, } \\
\text { and nasoendoscopic findings (polyp size) }\end{array}$ \\
\hline & & 12 & $\begin{array}{l}\text { CRSwNP without } H \text {. } \\
\text { pylori in nasal polyps }\end{array}$ & & & \\
\hline
\end{tabular}

a) CC: case-control; b) CRS: chronic rhinosinusitis; c) GORD: gastro-oesophageal reflux disease; d) FESS: functional endoscopic sinus surgery; e) QoL: quality of life; f) RSDI: rhinosinusitis disability index; g) SNOT-22: sinonasal outcome test-22; h) CSS: chronic sinusitis survey; i) CRSwNP: chronic rhinosinusitis with nasal polyps 
Table 5. Findings of included studies - studies of factors pathogenically linked to chronic rhinosinusitis (CRS).

\begin{tabular}{|c|c|c|c|}
\hline Study & Design & N (cases vs ${ }^{\mathrm{a}}$ controls) & Findings \\
\hline $\begin{array}{l}\text { Burduk, } \\
2011^{(54)}\end{array}$ & $\mathrm{CC}^{\mathrm{b}}$ & 20 vs 10 & $\begin{array}{l}\text { H. pylori DNA in all with } \mathrm{CRS}^{\mathrm{c}}(20 / 20) \text { and with normal nasal mucosa }(10 / 10) \text {. None had cagA } \\
\text { positive } H \text {. pylori detected. }\end{array}$ \\
\hline $\begin{array}{l}\text { Cvorovic, } \\
2008^{(26)}\end{array}$ & $\mathrm{CC}$ & 23 vs 15 & $\begin{array}{l}\text { H. pylori in } 6 / 23(26 \%) \text { with } C R S w N P^{d} \text { (by both rapid urease and histochemical testing); No } H \text {. } \\
\text { pylori in normal nasal mucosa }(0 / 15,0 \%) \text {. }\end{array}$ \\
\hline $\begin{array}{l}\text { Ozyurt, } \\
2009^{(55)}\end{array}$ & $\mathrm{CC}$ & 32 vs 27 & $\begin{array}{l}\text { H. pylori DNA in } 19 / 32(59 \%) \text { with CRSwNP and } 19 / 27 \text { (70\%) with normal nasal mucosa (not } \\
\text { statistically significant). In both groups, most } H \text {. pylori was cagA positive ( } 15 / 19 \text { vs } 17 / 19) \text {. }\end{array}$ \\
\hline $\begin{array}{l}\text { Dinis, } \\
2006^{(32)}\end{array}$ & $\mathrm{CC}$ & 15 vs 5 & $\begin{array}{l}\text { H. pylori DNA in } 6 / 15(40 \%) \text { with CRS and } 1 / 5(20 \%) \text { with normal nasal mucosa (not statistically } \\
\text { significant). }\end{array}$ \\
\hline $\begin{array}{l}\text { Kim, } \\
2007 \text { (56) }\end{array}$ & $\mathrm{CC}$ & 48 vs 29 & $\begin{array}{l}\text { H. pylori in } 12 / 48(25 \%) \text { with CRS and } 1 / 29(3.5 \%) \text { with normal nasal mucosa by both rapid } \\
\text { urease and histochemical testing (statistically significant). }\end{array}$ \\
\hline $\begin{array}{l}\text { Ozdek, } \\
2003^{(28)}\end{array}$ & $\mathrm{CC}$ & 12 vs 13 & H. pylori DNA in $4 / 12(33 \%)$ with CRS. No H. pylori in normal nasal mucosa $(0 / 13,0 \%)$ \\
\hline $\begin{array}{l}\text { Koc, } \\
2004^{(27)}\end{array}$ & $\mathrm{CC}$ & 30 vs 20 & $\begin{array}{l}\text { H. pylori in } 6 / 30(20 \%) \text { with nasal polyps by histochemical and serum antibody testing. No } H \text {. } \\
\text { pylori in normal nasal mucosa }(0 / 20,0 \%) \text {. }\end{array}$ \\
\hline $\begin{array}{l}\text { Nemati, } \\
2012^{(29)}\end{array}$ & $\mathrm{CC}$ & 25 vs 25 & No H. pylori in either population by $\mathrm{PCR}^{\mathrm{e}}, \mathrm{CLO}^{f}$ or culture. \\
\hline $\begin{array}{l}\text { Ozcan, } \\
2009^{(57)}\end{array}$ & $\mathrm{CC}$ & 25 vs 14 & $\begin{array}{l}\text { CLO test positive in } 1 / 25(4 \%) \text { with nasal polyps and } 2 / 14(14.3 \%) \text { with normal nasal mucosa. } \\
\text { Anti-H. pylori lgG }{ }^{9} \text { positive in } 6 / 25(24 \%) \text { vs } 3 / 14(21.4 \%) \text {. None had positive immunohistochemi- } \\
\text { cal staining or anti-H. pylori lgA } \mathrm{A}^{i} \text {. No differences were statistically significant. }\end{array}$ \\
\hline $\begin{array}{l}\text { Vceva, } \\
2012^{(46)}\end{array}$ & $\mathrm{CC}$ & 35 vs 30 & $\begin{array}{l}\text { Intranasal } H \text {. pylori PCR positive in significantly more subjects with nasal polyps }(10 / 35,28.6 \%) \\
\text { than normal nasal mucosa }(0 / 30,0 \%) \text {. Anti-H. pylori antibodies were found in significantly more } \\
\text { subjects with nasal polyps }(30 / 35,85.7 \%) \text { than normal nasal mucosa }(16 / 30,53.3 \%) \text {. All those } \\
\text { with positive PCR also had positive antibody testing. }\end{array}$ \\
\hline $\begin{array}{l}\text { DelGaudio, } \\
2005^{(39)}\end{array}$ & $\mathrm{CC}$ & 38 vs 10 vs 20 & $\begin{array}{l}\text { Nasopharyngeal reflux with } \mathrm{pH}<4 \text { significantly more prevalent with CRS }(15 / 38,39 \%) \text { than with } \\
\text { no CRS following FESS }(1 / 10,10 \%) \text { or no history of CRS }(2 / 20,10 \%) \text {. Nasopharyngeal reflux with } \\
\text { pH }<5 \text { significantly more common with CRS }(29 / 38,76 \%) \text { or no CRS following FESS }(5 / 10(50 \%) \\
\text { than with no history of CRS }(3 / 20,15 \%) \text {. Oesophageal reflux more prevalent with CRS }(25 / 38 \text {, } \\
66 \%) \text { than with no CRS following FESS }(3 / 10,30 \%) \text { or no history of CRS }(7 / 20,35 \%) \text {. }\end{array}$ \\
\hline $\begin{array}{l}\text { Ulualp, } \\
\text { 1999b }\end{array}$ & $\mathrm{CC}$ & 6 vs 12 vs 34 & $\begin{array}{l}\text { Hypopharyngeal reflux significantly more prevalent with CRS and LPRk }(4 / 6,67 \%) \text { than with CRS } \\
\text { alone }(4 / 12,34 \%) \text { or without CRS }(7 / 34,21 \%) \text {, the latter two not being significantly different. }\end{array}$ \\
\hline $\begin{array}{l}\text { Ulualp, } \\
\text { 1999a }\end{array}$ & $\mathrm{CC}$ & 11 vs 11 & $\begin{array}{l}\text { Hypopharyngeal reflux significantly more prevalent with CRS }(7 / 11,64 \%) \text { than without CRS } \\
(2 / 11,18 \%) \text {. Oesophageal reflux in all subjects, with significantly greater episode frequency and } \\
\text { overall acid exposure time in CRS. }\end{array}$ \\
\hline $\begin{array}{l}\text { Ozmen, } \\
2008^{(60)}\end{array}$ & $\mathrm{CC}$ & 33 vs 20 & $\begin{array}{l}\text { Hypopharyngeal reflux significantly more prevalent with CRS }(29 / 33,88 \%) \text { than without CRS } \\
(11 / 20,55 \%) \text {. Pepsin significantly more prevalent in nasal aspirates with CRS }(27 / 33,82 \%) \text {, than } \\
\text { without CRS }(10 / 20,50 \%) \text {. All with intranasal pepsin had hypopharyngeal reflux. }\end{array}$ \\
\hline $\begin{array}{l}\text { Jecker, } \\
2006^{(30)}\end{array}$ & $\mathrm{CC}$ & 20 vs 20 & $\begin{array}{l}\text { Significantly more GORD' with CRS (mean DeMeester Score } 32.9 \pm 8.7 \text { ) than without CRS (mean } \\
\text { DeMeester Score } 6.6 \pm 1.3 \text { ). Hypopharyngeal reflux not significantly different with or without } \\
\text { CRS. }\end{array}$ \\
\hline $\begin{array}{l}\text { Bhawana, } \\
2014^{(31)}\end{array}$ & $\mathrm{CC}$ & 50 vs 50 & $\begin{array}{l}\text { The middle meatus was more alkaline with } \mathrm{CRS}(\mathrm{pH} 7.81 \pm 0.83) \text { than without } \mathrm{CRS}(\mathrm{pH} \\
7.35 \pm 0.82) \text {. }\end{array}$ \\
\hline $\begin{array}{l}\text { Loehrl, } \\
2012^{(33)}\end{array}$ & $\mathrm{CC}$ & 5 vs 5 & $\begin{array}{l}\text { Intranasal pepsin in all nasal aspirates }(5 / 5) \text { with CRS, but none without CRS (0/5). Pepsin not } \\
\text { found in nasopharyngeal tissue of any CRS subjects }(0 / 5) \text {. }\end{array}$ \\
\hline $\begin{array}{l}\text { Dinis, } \\
2006^{(32)}\end{array}$ & $\mathrm{CC}$ & 15 vs 5 & $\begin{array}{l}\text { Pepsin and pepsinogen I concentrations in nasopharyngeal tissue not statistically different } \\
\text { with CRS or normal nasal mucosa. No tissue concentration exceeded the serum concentration; } \\
\text { average tissue:serum ratio was } 0.17 \text { for each. }\end{array}$ \\
\hline $\begin{array}{l}\text { Catalano, } \\
2004^{(61)}\end{array}$ & $\mathrm{CC}$ & 38 vs 117 & $\begin{array}{l}\text { Endoscopic or histological evidence of oesophagitis significantly more prevalent with CRS } \\
(11 / 38,29 \%) \text {, than without CRS }(18 / 117,15 \%) \text {. }\end{array}$ \\
\hline $\begin{array}{l}\text { Delehaye, } \\
2009^{(34)}\end{array}$ & $\mathrm{CC}$ & 37 vs 13 & $\begin{array}{l}\text { Saccharine clearance time greater with GORD }(23.79 \pm 5.58 \mathrm{~min}) \text { than with LPR }(8.15 \pm 2.06 \mathrm{~min}) \text {. } \\
\text { Mean SNOT-20n score significantly higher in GORD (19.3) than LPR (7.4). }\end{array}$ \\
\hline $\begin{array}{l}\text { Durmus, } \\
2010^{(35)}\end{array}$ & $\mathrm{CC}$ & 37 vs 30 & $\begin{array}{l}\text { No significant difference in saccharine clearance with GORD }(12.70 \pm 3.43 \mathrm{~min}) \text { or without GORD } \\
(13.11 \pm 3.33 \mathrm{~min}) \text {. No significant improvement in saccharine clearance time in GORD following } \\
\mathrm{PPI}^{\circ} \text { treatment }(13.10 \pm 3.34 \mathrm{~min}) \text {. }\end{array}$ \\
\hline $\begin{array}{l}\text { Wong, } \\
2010^{(36)}\end{array}$ & Cohort & 10 vs 10 & $\begin{array}{l}\text { No significant increase in nasal airflow resistance, nasal mucus production or sinonasal } \\
\text { symptoms following oesophageal infusion with saline or hydrochloric acid. }\end{array}$ \\
\hline
\end{tabular}

a) vs: versus; b) CC: case-control; c) CRS: chronic rhinosinusitis; d) CRSwNP: chronic rhinosinusitis with nasal polyps; e) PCR: polymerase chain reaction; f) CLO: campylobacterlike organism; g) IgG: immunoglobulin-G; h) CS: case series; i) IgA: immunoglobulin-A; j) FESS: functional endoscopic sinus surgery; k) LPR: laryngopharyngeal reflux; I) GORD: gastro-oesophageal reflux disease; m) RS: rhinosinusitis; n) SNOT-20: sino-nasal outcome test-20; o) PPI: proton-pump inhibitor 


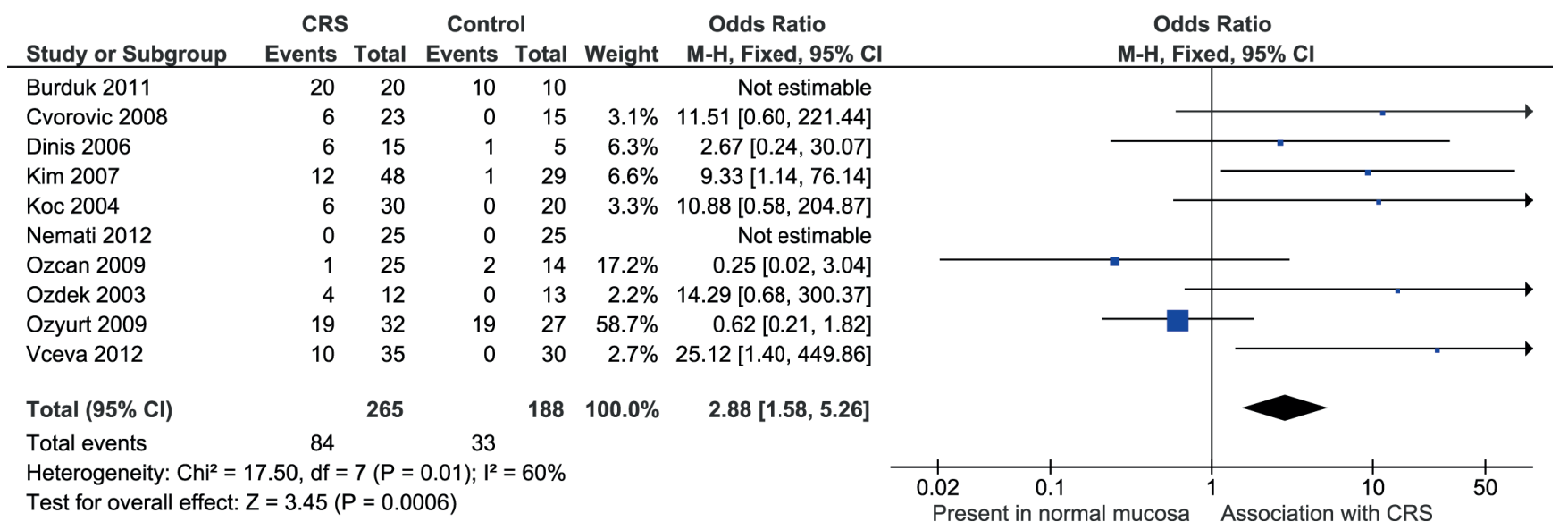

Figure 2. Odds ratio: H. pylori in the nasal cavity of groups with CRS (experimental) versus groups with normal nasal mucosa (control). M-H, MantelHaenszel.

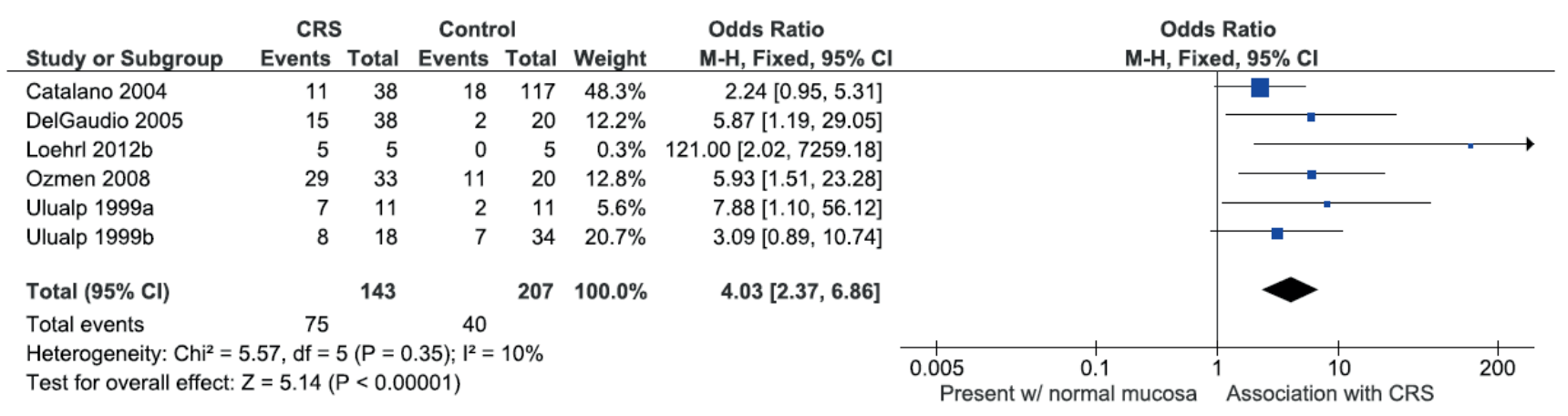

Figure 3. Odds ratio: Significant extra-gastric reflux in groups with CRS (experimental) versus groups without CRS (control). M-H, Mantel-Haenszel.

found that saccharine clearance time and SNOT-20 scores were greater in laryngopharyngeal reflux (LPR) than oesophageal reflux ${ }^{(34)}$; another found no difference in mucociliary clearance time with or without GORD ${ }^{(35)}$.

One study, by Wong ${ }^{(36)}$, assessed a neurological pathway contributing to CRS in reflux. This cohort study assessed nasal changes following saline or acid infusion into the oesophagus of subjects with no CRS or GORD. It did not find any significant differences in nasal airflow resistance, nasal mucus production or sinonasal symptoms following infusion of saline or hydrochloric acid.

\section{Epidemiological association}

The findings for studies of the epidemiological association between CRS and GORD are summarised in Table 6. These studies assessed the prevalence or incidence of CRS in a GORD population, or vice-versa. Meta-analysis of four case-control studies found CRS was more common in subjects with GORD $(3.15 \%, 3,270 / 103,919)$ than in control subjects without GORD $(1.83 \%, 2,121 / 115,751)$ (OR: 1.69 [1.60-1.79]) (Figure 4).

Similarly, meta-analysis of another two case-control studies, with 16,295 subjects, found that GORD was more prevalent in subjects with CRS $(29.6 \%, 2,403 / 8,142)$ than in control subjects without CRS (20.6\%, 1,680/8,153) (OR: 1.61 [1.50-1.73]) (Figure 5).

Two other case-control studies could not undergo meta-analysis, due to lack of relevant outcome data. Both showed greater prevalence of sinusitis in subjects with GORD than without GORD; one with OR 1.6 [1.2-2.0] ${ }^{(37)}$ and the other with increased mean SNOT-20 score among GORD subjects (22, 18-26 vs. 9 , 8-10)(38).

\section{Prognostic interaction between GORD and CRS}

These study findings are summarised in Table 7. The impact of GORD upon CRS severity following functional endoscopic sinus surgery (FESS) was the outcome of three case-control studies, while another similarly examined the impact of $\mathrm{H}$. pylori. DelGaudio found on $\mathrm{pH}$ testing that nasopharyngeal reflux was more common among those with persistent CRS than those with CRS resolution ${ }^{(39)}$. Similarly, Chambers found that those with persistent CRS were more likely to have a history of symptomatic GORD ${ }^{(40)}$. Deconde ${ }^{(41)}$ found similar subjective and objective sinus improvements following FESS in subjects with 
Table 6. Findings of included studies - epidemiological association between gastro-oesophageal Reflux (GORD) and chronic rhinosinusitis (CRS).

\begin{tabular}{|c|c|c|c|}
\hline Study & Design & $\begin{array}{l}\text { N (cases vs } \\
\text { controls) }\end{array}$ & Findings \\
\hline $\begin{array}{l}\text { Tan, } \\
2013^{(62)}\end{array}$ & $\mathrm{CC}^{\mathrm{b}}$ & $\begin{array}{l}595 \text { vs } 7523 \\
\text { vs } 8118\end{array}$ & $\begin{array}{l}\text { GORD prevalence significantly greater with CRSwNPd }(176 / 595,29.6 \%) \text { and CRSsNPe }(2220 / 7523, \\
29.0 \%) \text { than without CRS }(1666 / 8118,20.5 \%) . \text { The epidemiological association was statistically } \\
\text { significant (CRSwNP vs Controls: aORg 1.5 [1.2-1.8]; CRSsNP vs Controls: aOR } 1.7 \text { [1.6-1.8]). }\end{array}$ \\
\hline $\begin{array}{l}\text { Sedaghat, } \\
2012^{(63)}\end{array}$ & $\mathrm{CC}$ & 24 vs 35 & $\begin{array}{l}\text { GORD prevalence not significant different with } A R^{\mathrm{h}} \text { and subsequent } C R S(7 / 24,29.1 \%) \text { or with } A R \\
\text { alone }(14 / 35,40.0 \%) \text {. }\end{array}$ \\
\hline Ruhl, $2001^{(64)}$ & CC & 537 vs 6391 & RS' incidence not significantly different with GORD $(8 / 537,0.2 \%)$ or without GORD $(63 / 6391,0.1 \%)$. \\
\hline $\begin{array}{l}\text { Ruigomez, } \\
2004 \text { (37) }\end{array}$ & CC & $\begin{array}{l}7159 \text { vs } \\
10000\end{array}$ & $\begin{array}{l}\text { RS incidence significantly greater with GORD than without GORD (aOR: } 1.6 \text { [1.2-2.0]) in the year } \\
\text { following GORD diagnosis. }\end{array}$ \\
\hline $\begin{array}{l}\text { El-Serag, } \\
2001^{(65)}\end{array}$ & $\mathrm{CC}$ & $\begin{array}{l}1980 \text { vs } \\
7920\end{array}$ & $\begin{array}{l}\text { RS prevalence significantly greater with GORD }(83 / 1980,4.2 \%) \text {, than without GORD }(107 / 7920 \text {, } \\
1.4 \%) . \text { The epidemiological association was statistically significant (aOR } 2.3 \text { [1.7-3.2]). }\end{array}$ \\
\hline $\begin{array}{l}\text { El-Serag, } \\
1997^{(66)}\end{array}$ & $\mathrm{CC}$ & $\begin{array}{l}101366 \text { vs } \\
101366\end{array}$ & $\begin{array}{l}\text { RS prevalence significantly greater with GORD }(3165 / 101366,3.1 \%) \text {, than without GORD } \\
(1938 / 101366,1.9 \%) \text {. The epidemiological association was statistically significant (aOR 1.6 [1.5-1.7]). }\end{array}$ \\
\hline $\begin{array}{l}\text { Katle, } \\
2012^{(38)}\end{array}$ & $\mathrm{CC}$ & 77 vs 480 & $\begin{array}{l}\text { Mean SNOT-20j score significantly greater with GORD }(22 \pm 18.4, \mathrm{Cl}: 18-26) \text {, than among the general } \\
\text { population }(9 \pm 11.5, \mathrm{Cl}: 8-10) \text {. }\end{array}$ \\
\hline $\begin{array}{l}\text { Theodoropoulos, } \\
2001^{(49)}\end{array}$ & CC & 36 vs 74 & RS prevalence not significantly different with GORD (14/36, 39\%) or without GORD (13/74, 18\%). \\
\hline
\end{tabular}

a) vs: versus; b) CC: case-control; c) GORD: gastro-oesophageal reflux disease; d) CRSwNP: chronic rhinosinusitis with nasal polyps; e) CRSsNP: chronic rhinosinusitis without nasal polyps; f) CRS: chronic rhinosinusitis; g) aOR: adjusted odds ratio; h) AR: allergic rhinitis; i) RS: rhinosinusitis; j) SNOT-20: sino-nasal outcome test-20; k) CS: case series

Table 7. Findings of included studies - prognostic interaction between gastro-oesophageal reflux (GORD) and chronic rhinosinusitis (CRS).

\begin{tabular}{|c|c|c|c|}
\hline Study & Design & $\begin{array}{l}\text { N (cases vs } \\
\text { controls) }\end{array}$ & Findings \\
\hline $\begin{array}{l}\text { DelGaudio, } \\
2005^{(39)}\end{array}$ & $\mathrm{CC}^{\mathrm{b}}$ & $\begin{array}{l}38 \text { vs } 10 \\
\text { vs } 20\end{array}$ & $\begin{array}{l}\text { GORD }^{c} \text { significantly more prevalent with } \text { FESS }^{d} \text { resistant CRS=than with CRS resolved after FESS or with } \\
\text { no history of CRS. Nasopharyngeal reflux with } \mathrm{pH}<4 \text { among } 15 / 38(40 \%) \text { vs } 1 / 10(10 \%) \text { vs } 2 / 20 \text { (10\%). }\end{array}$ \\
\hline $\begin{array}{l}\text { Chambers, } \\
1997^{(40)}\end{array}$ & $\mathrm{CC}$ & 42 vs 140 & $\begin{array}{l}\text { GORD significantly more common with ongoing CRS symptoms after FESS (17/42, 41\%) than with CRS } \\
\text { resolved after FESS }(27 / 140,20 \%) \text {. }\end{array}$ \\
\hline $\begin{array}{l}\text { Deconde, } \\
2014^{(41)}\end{array}$ & $\mathrm{CC}$ & 72 vs 157 & $\begin{array}{l}\text { CRS improved significantly following FESS with or without GORD, with no significant difference. } \\
\text { Postoperatively, nasoendoscopic Lund \& Kennedy scores improved } 3.3 \pm 4.6 \text { vs } 4.4 \pm 4.2 \text { points; RSDI } \\
\text { improved } 22.7 \pm 22.1 \text { points vs } 22.2 \pm 21.4 \text { points; SNOT- } 22^{\mathrm{g}} \text { improved } 21.0 \pm 20.4 \text { points vs } 29.1 \pm 26.0 \\
\text { points; CSSh improved } 25.1 \pm 23.7 \text { points vs } 21.3 \pm 22.5 \text { points. }\end{array}$ \\
\hline $\begin{array}{l}\text { Jelavic, } \\
2012^{(42)}\end{array}$ & $\mathrm{CC}$ & 28 vs 12 & $\begin{array}{l}\text { Following FESS, nasoendoscopic findings of CRS improved significantly more with } \mathrm{H} \text {. pylori in nasal } \\
\text { polyps than without } \mathrm{H} \text {. pylori }(\mathrm{F}[1.38]=6.212, \mathrm{p}=0.017) \text { in nasal polyps. Symptomatic improvement } \\
\text { was not significantly different between groups }(\mathrm{F}[1.38]=1.881, \mathrm{p}=0.178) \text {. }\end{array}$ \\
\hline
\end{tabular}

a) vs: versus; b) CC: case-control; c) GORD: gastro-oesophageal reflux disease; d) FESS: functional endoscopic sinus surgery; e) CRS: chronic rhinosinusitis; f) RSDI: rhinosinusitis disability index; g) SNOT-22: sinonasal outcome test-22; h) CSS: chronic sinusitis survey

and without GORD. Jelavic ${ }^{(42)}$ found similar symptomatic sinus improvement following FESS in subjects with and without $\mathrm{H}$. pylori, while nasoendoscopic improvements were greater with H. pylori.

\section{Discussion}

Systematic review and meta-analysis of the current literature found that GORD has multiple associations with CRS. For control studies, these include an epidemiological association, rather than a response to GORD treatment. H. pylori is prevalent in sinonasal tissue with CRS. It is uncertain if these figures reflect the true prevalence, as there is no gold-standard test for $\mathrm{H}$. pylori ${ }^{(43)}$ and colonisation is reported as patchy in distribution, increasing the risk of false negative results ${ }^{\left({ }^{44)}\right.}$. A potentially pathogenic role of $H$. pylori in sinonasal inflammation has been suggested by previous authors ${ }^{(18,28)}$. Indeed, H. pylori is strongly pathogenic for gastric ulcers, gastritis and gastric cancers ${ }^{(45)}$. However, any pathogenic role remains uncertain and no studies to our knowledge have directly examined a potential mechanism in CRS. It is possible that the intranasal presence of $H$. pylori is not pathogenic, but is facilitated by pre-existing chronic inflammatory changes.

The intranasal presence of $H$. pylori may be a marker of nasopharyngeal reflux, or it may indicate that the nasal cavity is a reservoir for the bacteria. The current findings suggest that the 


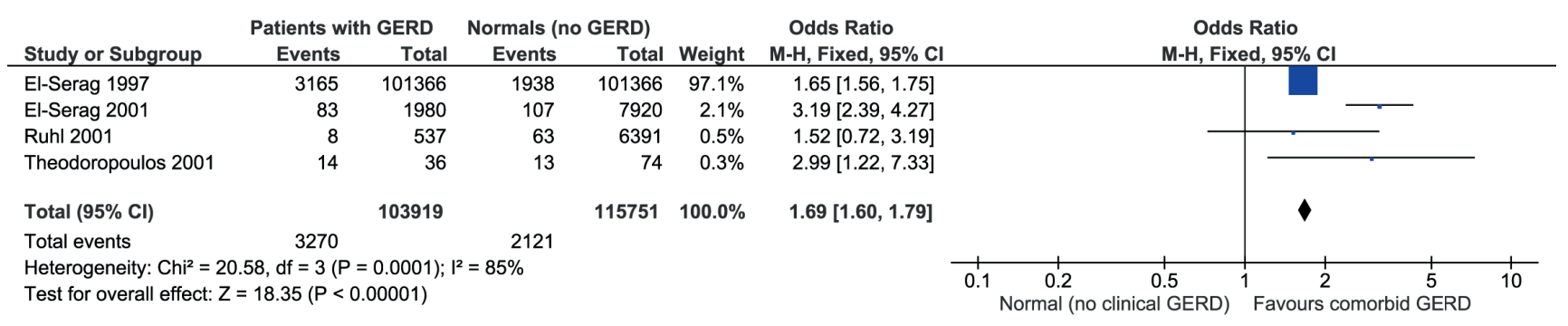

Figure 4. Odds ratio: Presence of CRS in groups with GORD (experimental) versus groups without GORD (control). M-H, Mantel-Haenszel.

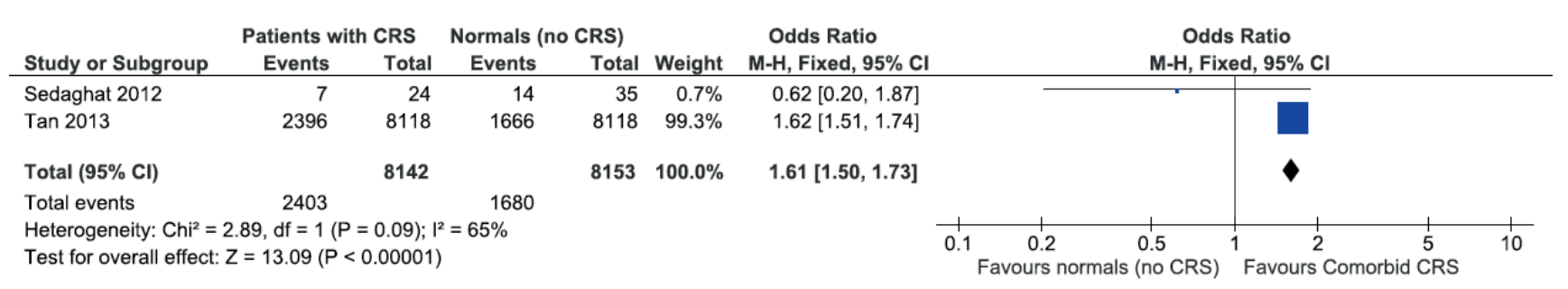

Figure 5. Odds ratio: Presence of GORD in groups with CRS (experimental) versus groups without CRS (control). M-H, Mantel-Haenszel.

stomach is the primary reservoir for $H$. pylori, which is supported by Vceva ${ }^{(46)}$ detecting $H$. pylori in the stomachs of all subjects, but in the nasal cavity of only some subjects.

Direct nasopharyngeal reflux is proposed as a pathogenic factor for CRS, through gastric acid and pepsin. Meta-analysis found that reflux is significantly more frequent with than without CRS. The nasal mucosa lacks the protective mechanisms present more distally against injury from reflux, which would lower the threshold for injury ${ }^{(39)}$. Standard $\mathrm{pH}$ study criteria typically consider a drop in $\mathrm{pH}$ below 4 to be pathologic, but even weakly acidic reflux may be pathologic in the proximal airway. The study of DelGaudio found that $\mathrm{pH}$ drops below 5 were more common in CRS than controls ${ }^{(39)}$ and multichannel impedance$\mathrm{pH}$ monitoring (MII-pH) has detected greater acid and non-acid reflux in CRS subjects than controls ${ }^{(47)}$.

Pepsin remains active at $\mathrm{pH}$ up to $7^{(48)}$ and has been found to contribute to lower airway pathology, so this may be involved even with normal or mildly deranged $\mathrm{pH}$ study results. Supporting this theory, pepsin was highly prevalent in sinonasal aspirates of CRS subjects, although it was not detected in sinonasal tissue biopsies. This indicates a potentially diagnostic role of the former test.

Indirect action of oesophageal refluxate on the upper airway has been proposed through a neurological pathway ${ }^{(19,49)}$. This has been demonstrated in the lower airways through pathways involving a hypervagal response ${ }^{(50,51)}$ and the release of tachykinin-like substances ${ }^{(52)}$. In rhinitis, decreased whole-body sympathetic function has been found with concomitant GORD
(53). It is believed that the sympathetic system increases nasal patency, while parasympathetic drive creates resistance and rhinorrhoea ${ }^{(53)}$. In this systematic review, a potential GORD driven neurological pathway was assessed by only one study. This did not find any significant sinonasal changes with acid or non-acid infusions into the oesophagus ${ }^{(36)}$. However, subjects did not have preceding GORD, so this did not examine the effects with chronic reflux exposure. Further research remains necessary before conclusions can be drawn.

Insufficient data also meant that no conclusions could be drawn about mucociliary function, with conflicting findings from the two relevant studies. It has been proposed that GORD delays mucociliary clearance, through inflammation from nasopharyngeal reflux or from a neurological pathway ${ }^{(34)}$. Yet further study is required.

Epidemiologically, the current meta-analysis and systematic review show an association of GORD with CRS. This reaffirms the common belief that prevalence of CRS is increased among GORD sufferers. However, this alone does not explain the nature of the association. The potentially pathogenic role of GORD was supported by Ruigomez ${ }^{(37)}$, who found increased incidence of sinusitis in the year after GORD diagnosis than among age and sex matched controls.

GORD has been proposed as a factor in failure of CRS treatment, which stands to reason if it carries an underlying pathogenic role. However, evidence surrounding the prognostic role of GORD in CRS is currently conflicting. Two studies did find that subjects with FESS failure were more likely to have GORD ${ }^{(39,40)}$. 
However, Deconde ${ }^{(41)}$ found FESS outcomes were not significantly different with or without GORD, and Jelavic ${ }^{(42)}$ found FESS outcomes were better in subjects with intranasal $H$. pylori than those without H. pylori.

The included studies were mainly case-controls, for which the compared study groups, or population, are intrinsically different. Studies in which bias assessment tools are used to identify differences between patients separated by interventions, such as randomised control trials or cohort studies, are nearly absent from the current literature. If a more defined CRS phenotype emerges, then such level 1 or 2 intervention studies might become available.

Heterogeneity of the included studies is a limitation of the current data, reflected by the high 12 values found on current meta-analysis. Differences in populations assessed are likely to have contributed. This is driven by factors such as the variable inclusion and exclusion criteria, disease definitions and their assessment, and inconsistent cessation of concurrent treatments. It restricts the depth of comparison, however, when the entire literature data was analysed collectively, the overall association remained significantly positive.

Additionally, exclusion of studies that did not specifically examine CRS may have limited the results. For example, studies of groups defined by the presence only of chronic cough or postnasal drip were excluded, as they are common and nonspecific symptoms, frequent beyond CRS. Restriction of the study to
English language publications may have caused an over-representation of certain racial groups.

\section{Conclusion}

There is a significant body of evidence demonstrating an association between GORD and CRS. While CRS is a multifactorial process, the evidence suggests that GORD does play a role in CRS, at least in some patients. Physicians should be cognizant of this and GORD should be considered as a factor, particularly in refractory CRS.

\section{Authorship contribution}

SRL: Data collection, analysis, manuscript production; HPB: Data collection, analysis, manuscript review; GO: Analysis, manuscript review; JR: Analysis, manuscript review; JMD: Design, expert manuscript review; JMC: Data collection, manuscript review; RS: Design, manuscript review; RJH: Design, analysis, manuscript review.

\section{Conflict of interest}

This is an unfunded project. Richard J Harvey is consultant with Medtronic, Olympus and NeilMed pharmaceuticals with research grant funding received from Meda Pharmaceuticals and Stallergenes. He has been on the speakers' bureau for GlaxoSmith-Kline and Arthrocare. Raymond Sacks is a consultant for Medtronic and Takeda Pharmaceuticals. Janet Rimmer has honoraria with Sanofi Aventis, Novartis, Mundipharma, BioCSL and Stallergenes. No other authors have any other disclosures to report.

\section{References}

1. Blackwell DL, Collins JG, Coles R. Summary health statistics for U.S. adults: National Health Interview Survey, 1997. Vital Health Stat 10. 2002; 205:1-109.

2. Chen $Y$, Dales R, Lin M. The epidemiology of chronic rhinosinusitis in Canadians. Laryngoscope. 2003;113(7):1199-205.

3. Collins JG. Prevalence of selected chronic conditions: United States, 1990-1992. Vital Health Stat 10. 1997; 194:1-89.

4. Shashy RG, Moore EJ, Weaver A. Prevalence of the chronic sinusitis diagnosis in Olmsted County, Minnesota. Arch Otolaryngol Head Neck Surg. 2004;130(3):320-3.

5. Vakil N, van Zanten SV, Kahrilas P, Dent J, Jones R, Global Consensus Group. The Montreal definition and classification of gastroesophageal reflux disease: a global evidence-based consensus. Am J Gastroenterol. 2006;101(8):1900-20.

6. El-Serag HB, Sweet S, Winchester CC, Dent J. Update on the epidemiology of gastrooesophageal reflux disease: a systematic review. Gut. 2014;63(6):871-80.

7. Wileman SM, McCann S, Grant AM, Krukowski ZH, Bruce J. Medical versus surgi- cal management for gastro-oesophageal reflux disease (GORD) in adults. Cochrane Database Syst Rev. 2010(3):CD003243.

8. Barbero GJ. Gastroesophageal reflux and upper airway disease. Otolaryngol Clin North Am. 1996;29(1):27-38.

9. Bothwell MR, Parsons DS, Talbot A, Barbero GJ, Wilder B. Outcome of reflux therapy on pediatric chronic sinusitis. Otolaryngol Head Neck Surg. 1999;121(3):255-62.

10. DiBaise JK, Huerter JV, Quigley EM. Sinusitis and gastroesophageal reflux disease. Ann Intern Med. 1998;129(12):1078.

11. DiBaise JK, Olusola BF, Huerter JV, Quigley EM. Role of GERD in chronic resistant sinusitis: a prospective, open label, pilot trial. Am J Gastroenterol. 2002;97(4):843-50,

12. Phipps CD, Wood WE, Gibson WS, Cochran WJ. Gastroesophageal reflux contributing to chronic sinus disease in children: a prospective analysis. Arch Otolaryngol Head Neck Surg. 2000;126(7):831-6.

13. Pincus RL, Kim HH, Silvers S, Gold S. A study of the link between gastric reflux and chronic sinusitis in adults. Ear Nose Throat J. 2006;85(3):174-8

14. Suskind DL, Zeringue GP 3rd, Kluka EA,
Udall J, Liu DC. Gastroesophageal reflux and pediatric otolaryngologic disease: the role of antireflux surgery. Arch Otolaryngol Head Neck Surg. 2001;127(5):511-4.

15. Bouchard S, Lallier M, Yazbeck S, Bensoussan A. The otolaryngologic manifestations of gastroesophageal reflux: when is a $\mathrm{pH}$ study indicated? J Pediatr Surg. 1999;34(7):1053-6.

16. Farhadi M, Noorbakhsh S, Tabatabaei A Searching the $\mathrm{H}$. pylori; serology\& PCR in children with adenoid hypertrophy and rhino sinusitis: A cross sectional study, Tehran, Iran. Med J Islam Repub Iran. 2013;27(2):77-82.

17. Monteiro VR, Sdepanian VL, Weckx L, Fagundes-Neto U, Morais MB. Twenty-fourhour esophageal $\mathrm{pH}$ monitoring in children and adolescents with chronic and/or recurrent rhinosinusitis. Braz J Med Biol Res. 2005;38(2):215-20

18. Morinaka S, Ichimiya M, Nakamura $H$. Detection of Helicobacter pylori in nasal and maxillary sinus specimens from patients with chronic sinusitis. Laryngoscope. 2003;113(9):1557-63.

19. Wong IW, Omari TI, Myers JC, et al. 
Nasopharyngeal pH monitoring in chronic sinusitis patients using a novel four channel probe. Laryngoscope. 2004;114(9):1582-5.

20. Filiaci F, Zambetti G, Luce M, Lo Vecchio A Docimo M, Romeo R. Research of non-specific hyperreactivity of upper airways in subjects with gastro-esophageal reflux (G.E.R.): preliminary reports. Allergol Immunopathol (Madr). 1997;25(6):266-71.

21. Nation J, Kaufman M, Allen M, Sheyn A, Coticchia J. Incidence of gastroesophageal reflux disease and positive maxillary antral cultures in children with symptoms of chronic rhinosinusitis. Int J Pediatr Otorhinolaryngol. 2014;78(2):218-22.

22. Pham V, Sykes K, Wei J. Long-term outcome of once daily nasal irrigation for the treatment of pediatric chronic rhinosinusitis Laryngoscope. 2014;124(4):1000-7.

23. Roka R, Rosztoczy A, Izbeki F, et al. Prevalence of respiratory symptoms and diseases associated with gastroesophageal reflux disease. Digestion. 2005;71 (2):92-6.

24. Yasaratne D, Madegedara D, Selvaratnam S, Kalukottage P, Rathnayake P, Gamage L. Epidemiology and aetiology of chronic rhinosinusitis in central Sri Lanka. Respirology. 2012;17:59.

25. Yellon RF, Coticchia J, Dixit S. Esophageal biopsy for the diagnosis of gastroesophageal reflux-associated otolaryngologic problems in children. Am J Med. 2000;108 Suppl 4a:131S-8S.

26. Cvorovic L, Brajovic D, Strbac M, Milutinovic Z, Cvorovic V. Detection of Helicobacter pylori in nasal polyps: preliminary report. J Otolaryngol Head Neck Surg. 2008;37(2):192-5.

27. Koc C, Arikan OK, Atasoy P, Aksoy A. Prevalence of Helicobacter pylori in patients with nasal polyps: a preliminary report. Laryngoscope. 2004;114(11):1941-4.

28. Ozdek A, Cirak MY, Samim E, Bayiz U, Safak MA, Turet S. A possible role of Helicobacter pylori in chronic rhinosinusitis: a preliminary report. Laryngoscope. 2003;113(4):679-82.

29. Nemati S, Mojtahedi A, Naghavi SE, Banan R, Zia F. Investigating Helicobacter pylori in nasal polyposis using polymerase chain reaction, urease test and culture. Eur Arch Otorhinolaryngol. 2012;269(5):1457-61.

30. Jecker P, Orloff LA, Wohlfeil M, Mann WJ. Gastroesophageal reflux disease (GERD), extraesophageal reflux (EER) and recurrent chronic rhinosinusitis. Eur Arch Otorhinolaryngol. 2006;263(7):664-7.

31. Bhawana GS, Kumar S, Kumar A. Alkaline $\mathrm{pH}$ in middle meatus in cases of chronic rhinosinusitis. Am J Otolaryngol. 2014;35(4):496-9.

32. Dinis PB, Subtil J. Helicobacter pylori and laryngopharyngeal reflux in chronic rhinosinusitis. Otolaryngol Head Neck Surg. 2006;134(1):67-72.

33. Loehrl TA, Samuels TL, Poetker DM, Toohill RJ, Blumin JH, Johnston N. The role of extraesophageal reflux in medically and surgically refractory rhinosinusitis. Laryngoscope. 2012;122(7):1425-30.
34. Delehaye E, Dore MP, Bozzo C, Mameli L, Delitala G, Meloni F. Correlation between nasal mucociliary clearance time and gastroesophageal reflux disease: our experience on 50 patients. Auris Nasus Larynx. 2009;36(2):157-61

35. Durmus R, Naiboglu B, Tek A, et al. Does reflux have an effect on nasal mucociliary transport? Acta Oto-Laryngol. 2010;130(9):1053-7.

36. Wong IW, Rees G, Greiff L, Myers JC, Jamieson GG, Wormald PJ. Gastroesophageal reflux disease and chronic sinusitis: in search of an esophageal-nasal reflex. Am J Rhinol Allergy. 2010;24(4):255-9.

37. Ruigomez A, Garcia Rodriguez LA, Wallander MA, Johansson S, Graffner $\mathrm{H}_{\text {, }}$ Dent J. Natural history of gastro-oesophageal reflux disease diagnosed in general practice. Aliment Pharmacol Ther. 2004;20(7):751-60.

38. Katle EJ, Hart H, Kjaergaard T, Kvaloy JT, Steinsvag SK. Nose- and sinus-related quality of life and GERD. Eur Arch Otorhinolaryngol. 2012;269(1):121-5.

39. DelGaudio JM. Direct nasopharyngeal reflux of gastric acid is a contributing factor in refractory chronic rhinosinusitis. Laryngoscope. 2005:115(6):946-57.

40. Chambers DW, Davis WE, Cook PR, Nishioka GJ, Rudman DT. Long-term outcome analysis of functional endoscopic sinus surgery: correlation of symptoms with endoscopic examination findings and potential prognostic variables. Laryngoscope. 1997;107(4):504-10.

41. Deconde AS, Mace JC, Smith TL. The impact of comorbid gastroesophageal reflux disease on endoscopic sinus surgery qualityof-life outcomes. Int Forum Allergy Rhinol. 2014;4(8):663-9

42. Jelavic B, Grgic M, Cupic H, Kordic M, Vasilj M, Baudoin T. Prognostic value of Helicobacter pylori sinonasal colonization for efficacy of endoscopic sinus surgery. Eur Arch Otorhinolaryngol. 2012;269(10):2197202.

43. Megraud F, Lehours P. Helicobacter pylori detection and antimicrobial susceptibility testing. Clin Microbiol Rev. 2007;20(2):280322.

44. Marshall BJ, Warren JR, Francis GJ, Langton SR, Goodwin CS, Blincow ED. Rapid urease test in the management of Campylobacter pyloridis-associated gastritis. Am J Gastroenterol. 1987;82(3):200-10.

45. Lee A, Fox J, Hazell S. Pathogenicity of Helicobacter pylori: a perspective. Infect Immun. 1993;61(5):1601-10.

46. Veeva A, Danic D, Vcev A, et al. The significance of Helicobacter pylori in patients with nasal polyposis. Med Glas (Zenica). 2012;9(2):281-6

47. Katle EJ, Hatlebakk JG, Grimstad T, et al., Gastro-oesophageal reflux in patients with chronic rhino-sinusitis investigated with multichannel impedance - $\mathrm{pH}$ monitoring. Rhinology. 2017; 55: 27-33.

48. Whitecross DP, Piper DW. The stability of human pepsins in stored gastric juice. Scand J Gastroenterol. 1975;10 (4):395-9.

49. Theodoropoulos DS, Ledford DK, Lockey RF, et al. Prevalence of upper respiratory symptoms in patients with symptomatic gastroesophageal reflux disease. Am J Respir Crit Care Med. 2001;164(1):72-6.

50. Harding SM, Richter JE. The role of gastroesophageal reflux in chronic cough and asthma. Chest. 1997;111(5):1389-402.

51. Lodi U, Harding SM, Coghlan HC, Guzzo MR, Walker LH. Autonomic regulation in asthmatics with gastroesophageal reflux. Chest. 1997;111(1):65-70.

52. Hamamoto J, Kohrogi $\mathrm{H}$, Kawano O, et al. Esophageal stimulation by hydrochloric acid causes neurogenic inflammation in the airways in guinea pigs. J Appl Physiol. 1997;82(3):738-45.

53. Loehrl TA, Smith TL, Darling RJ, et al Autonomic dysfunction, vasomotor rhinitis, and extraesophageal manifestations of gastroesophageal reflux. Otolaryngol Head Neck Surg. 2002;126(4):382-7.

54. Burduk PK, Kaczmarek A, Budzynska A, Kazmierczak W, Gospodarek E. Detection of Helicobacter pylori and cagA gene in nasal polyps and benign laryngeal diseases. Arch Med Res. 2011:42(8):686-9.

55. Ozyurt M, Gungor A, Ergunay K, Cekin E, Erkul E, Haznedaroglu T. Real-time PCR detection of Helicobacter pylori and virulence-associated cagA in nasal polyps and laryngeal disorders. Otolaryngol Head Neck Surg. 2009;141(1):131-5.

56. Kim HY, Dhong HJ, Chung SK, Chung KW, Chung YJ, Jang KT. Intranasal Helicobacter pylori colonization does not correlate with the severity of chronic rhinosinusitis. Otolaryngol Head Neck Surg. 2007;136(3):390-5.

57. Ozcan C, Polat A, Otag F, Gorur K. Does Helicobacter pylori play a role in etiology of nasal polyposis? Auris Nasus Larynx. 2009;36(4):427-30

58. Ulualp SO, Toohill RJ, Shaker R. Pharyngeal acid reflux in patients with single and multiple otolaryngologic disorders. Otolaryngol Head Neck Surg. 1999;121(6):725-30.

59. Ulualp SO, Toohill RJ, Hoffmann R, Shaker R. Possible relationship of gastroesophagopharyngeal acid reflux with pathogenesis of chronic sinusitis. Am J Rhinol. 1999:13(3):197-202.

60. Ozmen S, Yucel OT, Sinici I, et al. Nasal pepsin assay and $\mathrm{pH}$ monitoring in chronic rhinosinusitis. Laryngoscope. 2008;1 18(5):8904.

61. Catalano F, Terminella C, Grillo C, Biondi S, Zappala M, Bentivegna C. Prevalence of oesophagitis in patients with persistent upper respiratory symptoms. J Laryngol Otol. 2004;118(11):857-61.

62. Tan BK, Chandra RK, Pollak J, et al. Incidence and associated premorbid diagnoses of patients with chronic rhinosinusitis. J Allergy Clin Immunol. 2013;131(5):1350-60.

63. Sedaghat AR, Gray ST, Wilke CO, Caradonna DS. Risk factors for development of chronic 
rhinosinusitis in patients with allergic rhinitis. Int Forum Allergy Rhinol. 2012;2(5):3705.

64. Ruhl CE, Sonnenberg A, Everhart JE. Hospitalization with respiratory disease following hiatal hernia and reflux esophagitis in a prospective, population-based study. Ann Epidemiol. 2001;11(7):477-83.

65. El-Serag HB, Gilger M, Kuebeler M Rabeneck L. Extraesophageal associations of gastroesophageal reflux disease in children without neurologic defects. Gastroenterology. 2001;121(6):1294-9.

66. El-Serag HB, Sonnenberg A. Comorbid occurrence of laryngeal or pulmonary disease with esophagitis in United States military veterans. Gastroenterology. 1997;113(3):755-60.

Stewart Leason

Ground Floor

67 Burton St

Darlinghurst NSW 2010

Australia

E-mail: stewartleason@gmail.com
ADVERTISEMENT

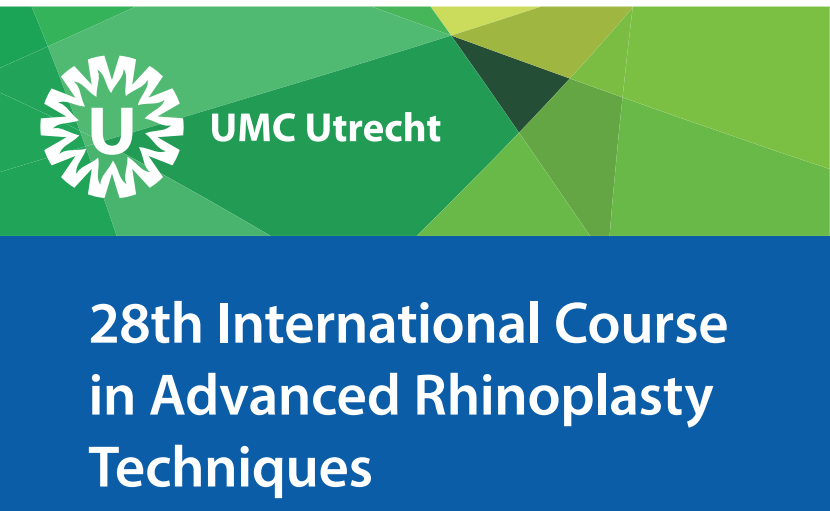

18 - 20 October, 2017

\section{UMC Utrecht, The Netherlands}

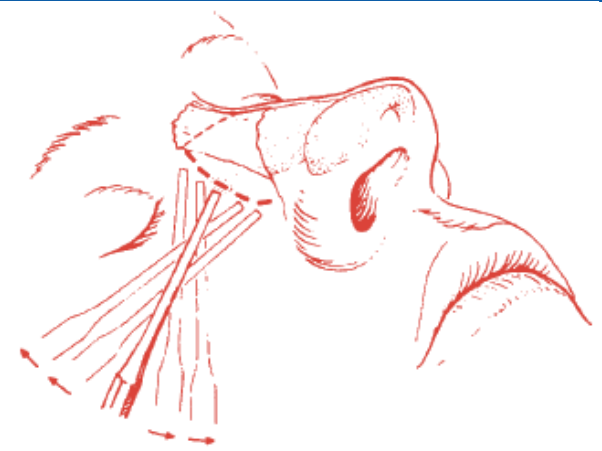

Course Directors: D.J. Menger \& G.J. Nolst Trenité

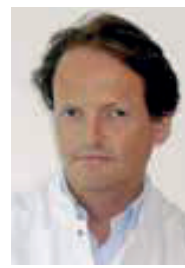

Dirk Jan Menger

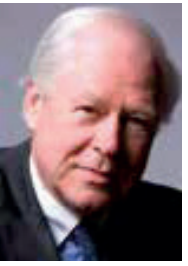

Gilbert Nolst Trenité

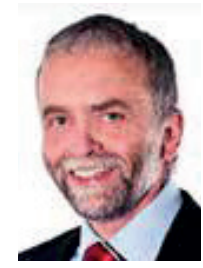

W. Gubisch
Teacher of honour: Wolfgang Gubisch, Stuttgart, Germany

- multidisciplinary international faculty

- didactic lectures and expert panels

- live surgery

- fresh frozen cadaver dissection of the Nose

-Workshop Otoplasty and auricular reconstruction

Preliminary facultry:

-F. Apaydin

- N. Janssen

- M. Kon

-1. Ligtenberg

-M. Bönisch

•I. Ligtenberg
•P.J.F.M. Lohuis

- Tj.D. Bruintjes

- M.H.J.M. Majoor

- D.J. Menger
- M.J. Middelweerd

-W. Gubisch

-M. Muradin

F.W.A. Otten

- A. Tasman

- H.D. Vuyk

$\cdot$ K.J.A.O. Ingels

-C.C. Wever

Information: rhinoplasty@umcutrecht.nl

website: www.advancedrhinoplasty.nl

sums 